\title{
Symmetric multiple chessboard complexes and a new theorem of Tverberg type
}

\author{
Duško Jojić \\ Faculty of Science \\ University of Banja Luka
}

\author{
Siniša T. Vrećica* \\ Faculty of Mathematics \\ University of Belgrade
}

August 14, 2016

\author{
Rade T. Živaljević* \\ Mathematical Institute \\ SASA, Belgrade
}

\begin{abstract}
We prove a new theorem of Tverberg-van Kampen-Flores type (Theorem 1.2) which confirms the conjecture of Blagojević, Frick, and Ziegler about the existence of 'balanced Tverberg partitions' (Conjecture 6.6 in, Tverberg plus constraints, Bull. London Math. Soc. 46 (2014) 953-967). The conditions in Theorem 1.2 are somewhat weaker than in the original conjecture and we show that the theorem is optimal in the sense that the new (weakened) condition is also necessary. Among the consequences is a positive answer (Theorem 7.2) to the 'balanced case' of the problem whether each admissible $r$-tuple is Tverberg prescribable, [BFZ, Question 6.9].
\end{abstract}

\section{Introduction}

Multiple chessboard complexes are studied in their own right [KRW, JVZ-1] as interesting graph complexes where (in the spirit of [J08]) the analysis of connectivity properties is one of the central themes.

The relevance of general chessboard complexes for theorems of Tverberg type is well known [BMZ, M03, VŽ94, ŽV92, Zi11, Ž04]. Perhaps it should not come as a surprise, as anticipated already in [JVZ-1], that multiple chessboard complexes are not an exception and that they should also quite naturally arise in this context.

In this paper we demonstrate that the symmetric multiple chessboard complexes (symmetrized versions of multiple chessboard complexes) are indeed natural configuration spaces for the proof of new results of Tverberg type.

Our central new results are Theorems 1.2 and 7.2 . Theorem 1.2 provides an affirmative answer to the following conjecture of Blagojević, Frick, and Ziegler, [BFZ].

*Supported by the Ministry for Science and Technology of Serbia, Grant 174034. 
Conjecture $1.1([\mathrm{BFZ}$, Conjecture 6.6.]) Let $r \geq 2$ be a prime power, $d \geq 1, N \geq$ $(r-1)(d+2)$, and $r(k+1)+s>N+1$ for integers $k \geq 0$ and $0 \leq s<r$. Then, for every continuous map $f: \Delta_{N} \rightarrow \mathbb{R}^{d}$, there are $r$ pairwise disjoint faces $\sigma_{1}, \ldots, \sigma_{r}$ of $\Delta_{N}$ such that $f\left(\sigma_{1}\right) \cap \cdots \cap f\left(\sigma_{r}\right) \neq \emptyset$, with dim $\sigma_{i} \leq k+1$ for $1 \leq i \leq s$ and dim $\sigma_{i} \leq k$ for $s<i \leq r$.

Theorem 1.2 establishes the conjecture in full generality. Moreover, it improves the conjecture in the sense that the condition $r(k+1)+s>N+1$ in Conjecture 1.1 is replaced in Theorem 1.2 by a weaker and possibly more natural condition $r k+s \geq$ $(r-1) d$. This condition is indeed weaker since, by assuming both conditions from Conjecture 1.1, we have the inequalities,

$$
r k+s>N+1-r \geq(r-1)(d+2)-(r-1)=(r-1)(d+1)>(r-1) d .
$$

Observe that the condition $r k+s \geq(r-1) d$ is also necessary. Indeed it expresses the fact that if for a generic affine map $f$ the intersection $\cap_{i=1}^{r} f\left(\sigma_{i}\right)$ is non-empty then,

$$
\operatorname{codim}\left(f\left(\sigma_{1}\right)\right)+\ldots+\operatorname{codim}\left(f\left(\sigma_{r}\right)\right)=\operatorname{codim}\left(\cap_{i=1}^{r} f\left(\sigma_{i}\right)\right) \leq d .
$$

Theorem 1.2 Let $r \geq 2$ be a prime power, $d \geq 1, N \geq(r-1)(d+2)$, and $r k+s \geq$ $(r-1) d$ for integers $k \geq 0$ and $0 \leq s<r$. Then for every continuous map $f: \Delta_{N} \rightarrow \mathbb{R}^{d}$, there are $r$ pairwise disjoint faces $\sigma_{1}, \ldots, \sigma_{r}$ of $\Delta_{N}$ such that $f\left(\sigma_{1}\right) \cap \cdots \cap f\left(\sigma_{r}\right) \neq \emptyset$, with $\operatorname{dim} \sigma_{i} \leq k+1$ for $1 \leq i \leq s$ and $\operatorname{dim} \sigma_{i} \leq k$ for $s<i \leq r$.

The proof of Theorem 1.2 is given in Section 5. It relies on the shellability of the associated configuration space (symmetric, multiple chessboard complex), which is established in Section 4. As a consequence we obtain a fundamental connectivity result for these complexes (Theorem 3.3 in Section 3). The introduction of 'symmetric multiple chessboard complexes' (Section 2.1) is one of key new ideas used in the proof of Theorem 1.2, For this reason a more general construction of 'symmetrized deleted joins' is reviewed in Section 2.2.

\subsection{Some consequences and relatives of Theorem 1.2}

Theorem 1.2 is apparently not an immediate consequence of any other known result of Tverberg type (see Section 1.2 for a brief discussion). This observation opens the question of the relative strength of Theorem 1.2 and serves as a motivation for the systematic study of the associated reduction methods based on Sarkaria's inequality (Sections 6] and 7).

Theorem 1.2 is a candidate for the 'to date' most general known result of van Kampen-Flores type. Here is a list of some of its immediate consequences.

(1) It provides an optimal positive answer (see Theorem 7.2 in Section 7) to the 'balanced case' of the problem whether each admissible $r$-tuple is Tverberg prescribable [BFZ, Question 6.9]; 
(2) The classical van Kampen-Flores theorem (see [M03, Theorem 5.1.1] or [BFZ, Theorem 6.1]) is obtained if $d$ is even, $r=2, s=0$, and $k=\frac{d}{2}$;

(3) The sharpened van Kampen-Flores theorem [BFZ, Theorem 6.8] corresponds to the case when $d$ is odd, $r=2, s=1$, and $k=\left\lfloor\frac{d}{2}\right\rfloor$;

(4) The case $d=3$ of the 'sharpened van Kampen-Flores theorem' is following [BFZ, Section 6] equivalent to the Conway-Gordon-Sachs theorem which says that the complete graph $K_{6}$ on 6 vertices is 'intrinsically linked';

(5) The generalized van Kampen-Flores theorem [BFZ, Theorem 6.3], which improves upon the results of Sarkaria [Sar, Section 1.5.] and Volovikov [Vol-2], follows for $s=0$ and $k=\left\lceil\frac{r-1}{r} d\right\rceil$.

We have already seen that the condition $r k+s \geq(r-1) d$ in Theorem 1.2 cannot be improved (see inequalities (11) and (2) ). The other condition $N \geq(r-1)(d+2)$ (in the case $k+1<d)$ is also tight, as shown essentially by the example [Sar, Section 1.5.], see also [BFZ, Section 6] for a related discussion.

Theorem 1.2 can be extended to maps from the simplex $\Delta_{N}$ to $d$-manifolds, following the scheme proposed by Volovikov in [Vol-1] (see also [BFZ, Section 6]). This extension of Theorem 1.2 does not require new ideas so we omit the details.

\subsection{Proof methods and techniques}

There have been two main general proof techniques used in topological (nonlinear) problems of Tverberg-van Kampen-Flores type. These two proof schemes may be informally referred to as the 'direct' and 'indirect' proof methods.

(1) The 'direct methods' rely on a variant of equivariant obstruction theory and can be classified as:

(1a) the methods which use the high connectivity of the configuration space;

(1b) the methods involving a direct calculation of the obstruction.

(2) The 'indirect methods' comprise two basic form of reductions:

(2a) the 'constraint method' or the Gromov-Blagojević-Frick-Ziegler reduction;

(2b) the methods based on 'Sarkaria's index inequality' and its relatives.

Examples of the 'direct approach' include the proof of the 'Topological Tverberg theorem' BSS and the 'Type B colored Tverberg theorem' VŽ94 (both results are classified as (1a)). The results illustrating (1b) proof scheme are the 'Generalized van Kampen-Flores theorem' [Vol-2], the 'Optimal colored Tverberg theorem' [BMZ], [VŽ11], and (again) the 'Topological Tverberg theorem' [Öz87]. 
The proof of Theorem 1.2 is also direct (it is classified as the (1a) type). It relies on the shellability of the associated symmetric multiple chessboard complex and the well known fact that pure $n$-dimensional shellable complexes are $(n-1)$-connected.

The elegant and powerful 'constraint method' [BFZ], known also as the GromovBlagojević-Frick-Ziegler reduction, was instrumental in the recent spectacular construction of counterexamples for the general topological Tverberg conjecture, see [BBZ] for the exposition of the history of the problem. According to the diagram of implications on page 2 in [BZ], all known (topological) results of Tverberg-van Kampen-Flores type are reducible by the 'constraint method' to either the Topological Tverberg Theorem (TTT) or the Optimal Colored Tverberg Theorem (OCTT).

Theorem 1.2 is apparently the first result which is not an immediate consequence of either (TTT) or (OCTT).

For this reason it may be interesting to (1) explore the nature of this phenomenon and (2) determine which results can be reduced to Theorem 1.2, by either the 'constraint method' or some other reduction procedure. With these objectives in mind we develop in Section 6 some new type (2b) reductions (based on Sarkaria's index inequality) which appear to be better adapted to the use of 'symmetrized deleted joins' than the original 'constraint method'.

\subsection{Admissible, prescribable and persistent partitions}

Theorem 1.2 is an example of a Tverberg type result where one can prescribe in advance (as in the classical van Kampen - Flores theorem) an upper bound $\operatorname{dim}\left(\sigma_{i}\right) \leq d_{i}$ on the dimension of simplices in a Tverberg $r$-partition for any map $f: \Delta_{N} \rightarrow \mathbb{R}^{d}$.

There is a general and a very interesting problem (Question 6.9. in [BFZ]) asking for a characterization of $r$-tuples $\left(d_{i}\right)_{i=1}^{r}$ which are 'Tverberg prescribable' (see [BFZ, Definition 6.7] or our Definition [7.1).

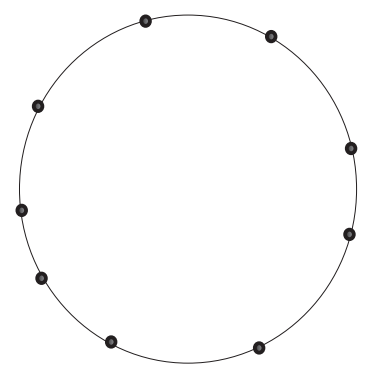

(a)

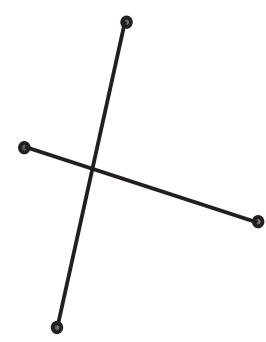

(b)

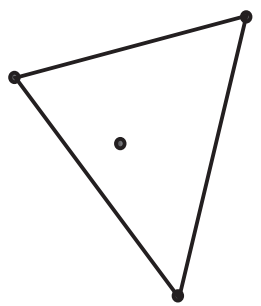

(c)

Figure 1: In Radon's theorem the $(2,2)$-partitions are persistent, while $(3,1)$ are not.

The essence of the problem is nicely illustrated already by the planar case of Radon's theorem, Figure 1. Indeed, each collection $S \subset \mathbb{R}^{2}$ of four points in the plane admits a partition $S=S_{1} \uplus S_{2}$ such that $\operatorname{conv}\left(S_{1}\right) \cap \operatorname{conv}\left(S_{2}\right) \neq \emptyset$. 
Depending on the size of the sets $S_{i}$ there are two types of partitions, a $(2,2)$ partition, as in Figure 1 (b), or a (3,1)-partition, as in Figure 1 (c). Note however that these two types of partitions are not necessarily equally represented if $S$ has 5 points or more. Indeed, the $(2,2)$-configurations are always present in such a set (we may also say that $(2,2)$-partitions are persistent) while $(3,1)$-configuration are not persistent, for example they never appear if $S$ is in convex position, Figure 1 (a). In the language of [BFZ] (Definition 6.7) the dimension pair $\left(d_{1}, d_{2}\right)=(1,1)$ (corresponding to $(2,2)$ partitions) is Tverberg prescribable while the pair $\left(d_{1}, d_{2}\right)=(2,0)$ is not!

As a consequence of Theorem 1.2 we establish a result (Theorem 7.2) which addresses the case where the prescribed bounds $d_{i}$ are 'balanced' in the sense that $\left|d_{i}-d_{j}\right| \leq 1$ for each $i$ and $j$.

Theorem 7.2, as an instance of the general A-P conjecture (Section 7), is certainly of some independent interest so we include in Section 7 an alternative approach to this result, illustrating the 'extended Sarkaria's reduction' (Section 6.3).

\section{Symmetric configuration spaces}

A large symmetry group of the configuration space is one of the key ingredients in the usual configuration space/test map-scheme [Ž04] for applying topology to problems of discrete geometry. If our first choice for the configuration space is not sufficiently symmetric, it is quite natural to introduce a bigger space which admits a (fixed point) free group action.

\subsection{Symmetric multiple chessboard complexes}

Suppose that $\mathbf{k}=\left(k_{i}\right)_{i=1}^{n}$ and $\mathbf{p}=\left(p_{j}\right)_{j=1}^{m}$ are two sequences of non-negative integers. The associated multiple chessboard complex

$$
\Delta_{m, n}^{\mathbf{k}, \mathbf{p}}=\Delta_{m, n}^{k_{1}, \ldots, k_{n} ; p_{1}, \ldots, p_{m}}
$$

is following [KRW, JVZ-1] defined as the complex of all rook-placements $A \subset[m] \times[n]$ such that at most $k_{i}$ rooks are allowed to be in the $i$-th row (for $i=1, \ldots, n$ ), and at most $p_{j}$ rooks are allowed in the $j$-th column (for $j=1, \ldots, m$ ). As in [JVZ-1] we pay special attention to the complexes $\Delta_{m, n}^{\mathbf{k}, \mathbf{1}}=\Delta_{m, n}^{k_{1}, \ldots, k_{n} ; \mathbf{1}}$ where $p_{1}=\ldots=p_{m}=1$.

Let $G \subset S_{n}$ be a subgroup of the symmetric group $S_{n}$ acting on the set of rows of the chessboard $[m] \times[n]$. The multiple chessboard complex $\Delta_{m, n}^{k_{1}, \ldots, k_{n} ; \mathbf{1}}$ is rarely $G$ invariant. Since the $G$-invariance of the configuration space is an essential feature of the usual 'configuration space/test map - scheme' [Živ98, Ž04], it is quite natural to define a symmetric version of $\Delta_{m, n}^{k_{1}, \ldots, k_{n} ; \mathbf{1}}$.

Definition 2.1 The G-symmetric multiple chessboard complex

$$
\Sigma\left(\Delta_{m, n}^{\mathbf{k}, \mathbf{1}} ; G\right)=\Sigma\left(\Delta_{m, n}^{k_{1}, \ldots, k_{n} ; \mathbf{1}} ; G\right)=\bigcup_{g \in G} \Delta_{m, n}^{k_{g(1)}, \ldots, k_{g(n)} ; \mathbf{1}}
$$


is obtained from the multiple chessboard complex $\Delta_{m, n}^{k_{1}, \ldots, k_{n} ; \mathbf{1}}$ by the symmetrization with respect to $G$. In the special case $G=S_{n}$ we obtain the complex,

$$
\Sigma_{m, n}^{\mathbf{k}, \mathbf{1}}=\Sigma_{m, n}^{k_{1}, \ldots, k_{n} ; \mathbf{1}}=\Sigma\left(\Delta_{m, n}^{k_{1}, \ldots, k_{n} ; \mathbf{1}}\right):=\Sigma\left(\Delta_{m, n}^{k_{1}, \ldots, k_{n} ; \mathbf{1}} ; S_{n}\right)
$$

which is referred to as the symmetric multiple chessboard complex.

Remark 2.2 If one starts with more general multiple chessboard complexes $\Delta_{m, n}^{\mathbf{k}, \mathbf{p}}=$ $\Delta_{m, n}^{k_{1}, \ldots, k_{n} ; p_{1}, \ldots, p_{m}}$ it may be sometimes more natural to symmetrize with respect to the group $G=H \times Q$ where $H$ permutes the rows and $Q$ permutes the columns of the chessboard $[m] \times[n]$. In this paper we do not need these more general complexes.

\subsection{Symmetrized deleted joins}

The 'symmetric multiple chessboard complex' $\Sigma\left(\Delta_{m, n}^{k_{1}, \ldots, k_{n} ; \mathbf{1}}\right)$, described in Definition 2.1, exemplifies a much more general 'symmetrized deleted join' construction.

Definition 2.3 Let $\mathcal{K}=\left\langle K_{1}, \ldots, K_{n}\right\rangle$ be a collection of not necessarily distinct simplicial complexes $K_{i} \subset 2^{[m]}=\Delta([m])$. Let $\mathcal{K}_{\Delta}^{* n}=K_{1} *_{\Delta} \ldots *_{\Delta} K_{n} \subset\left(\Delta([m])_{\Delta}^{* n}\right) \cong[n]^{* m}$ be the associated deleted join. The complex obtained from $\mathcal{K}_{\Delta}^{* n}$ by the $S_{n}$-symmetrization,

$$
\Sigma\left(\mathcal{K}_{\Delta}^{* n}\right):=\bigcup_{\pi \in S_{n}} K_{\pi(1)} *_{\Delta} \ldots *_{\Delta} K_{\pi(n)} \subset[n]^{* m}
$$

is referred to as the symmetrized deleted join of $\mathcal{K}$.

Definition 2.4 Let $\mathcal{K}=\left\langle K_{1}, \ldots, K_{n}\right\rangle$ be an ordered collection of simplicial subcomplexes of $2^{[m]}=\Delta([m])$. Let $a=\left(A_{1}, \ldots, A_{n}\right)$ be a pairwise disjoint family of subsets of $[m]$, describing a simplex in $\Delta([m])_{\Delta}^{* n}$. The associated $\in$-graph,

$$
\Gamma_{a}^{\epsilon}=\Gamma_{a, \mathcal{K}}^{\epsilon}=\left\{(i, j) \in[n] \times[n] \mid A_{i} \in K_{j}\right\}
$$

is referred to as the 'epsilon graph' of a and $\mathcal{K}$ (or of a alone if $\mathcal{K}$ is fixed and clear from the context).

The following proposition (relevant for Sections 6.2 and 6.3) provides a simple criterion for an $n$-tuple $a=\left(A_{1}, \ldots, A_{n}\right) \in[n]^{* m}$ to be an element of $\Sigma\left(\mathcal{K}_{\Delta}^{* n}\right)$.

Proposition 2.5 A simplex $a=\left(A_{1}, \ldots, A_{n}\right) \in[n]^{* m} \in[n]^{* m}$ belongs to $\Sigma\left(\mathcal{K}_{\Delta}^{* n}\right)$ if and only if the associated $\in$-graph $\Gamma_{a}^{\in}$ admits a complete (perfect) matching.

The bipartite graph $\Gamma_{a}^{\notin}=\left\{(i, j) \in[n] \times[n] \mid A_{i} \notin K_{j}\right\}$, complementing $\Gamma_{a}^{\in}$ in the complete bipartite graph $K_{n, n}$, is referred to as the 'non- $\in$ graph' of $a$. The following proposition illustrates the role of non- $\in$ graphs in the definition of 'collective $n$-unavoidable complexes', introduced in [JNPZ]. 
Proposition 2.6 A collection $\mathcal{K}=\left\langle K_{1}, \ldots, K_{n}\right\rangle$ of simplicial subcomplexes of $2^{[m]}=$ $\Delta([m])$ is 'collective $n$-unavoidable', [JNPZ, Definition 3.1], if and only if for each simplex $a=\left(A_{1}, \ldots, A_{n}\right) \in[n]^{* m}$ the associated non- $\in$ graph $\Gamma_{a}^{\notin}$ does NOT admit a complete matching.

Proof: By [JNPZ, Definition 3.1] a collection $\mathcal{K}=\left\langle K_{1}, \ldots, K_{n}\right\rangle$ is 'collective $n$ unavoidable' if for each (ordered) partition $B_{1} \uplus \ldots \uplus B_{n}=[\mathrm{m}]$ of $[\mathrm{m}]$ there exists $i \in[n]$ such that $B_{i} \in K_{i}$. This condition is easily seen to be equivalent to the condition that the associated non- $\in$ graph does not have a perfect matching.

\section{The connectivity of $\sum_{m, n}^{\mathrm{k}, 1}$}

One of the main results of [JVZ-1] is a lower bound for the connectivity of the multiple chessboard complex $\Delta_{m, n}^{\mathbf{k}, \mathbf{1}}=\Delta_{m, n}^{k_{1}, \ldots, k_{n} ; \mathbf{1}}$.

Theorem 3.1 ([JVZ-1, Theorem 3.1.]) The generalized chessboard complex $\Delta_{m, n}^{k_{1}, \ldots, k_{n} ; \mathbf{1}}$ is $\mu$-connected where,

$$
\mu=\min \left\{m-n-1, k_{1}+\cdots+k_{n}-2\right\}
$$

In particular if $m \geq k_{1}+\cdots+k_{n}+n-1$ then $\Delta_{m, n}^{k_{1}, \ldots, k_{n} ; \mathbf{1}}$ is $\left(k_{1}+\cdots+k_{n}-2\right)$-connected.

Remark 3.2 As remarked already in JVZ-1 the estimate $\mu \geq m-n-1$ in (7) can be significantly improved for small values of $m$. However, as in [JVZ-1, we are here mainly interested in the values of $m$ for which the complex $\Delta_{m, n}^{k_{1}, \ldots, k_{n} ; \mathbf{1}}$ is $\left(k_{1}+\cdots+k_{n}-2\right)$ connected since this is precisely the result used in applications to generalized Tverberg theorems. For this reason our working hypothesis will be most of the time the inequality,

$$
m \geq k_{1}+\cdots+k_{n}+n-1
$$

A new approach to this (central) case of Theorem 3.1, based on discrete Morse theory, is developed in [JNPZ.

Motivated by Conjecture 6.6. from [BFZ] (Conjecture 1.1), we focus our attention to the multiple chessboard complex $K_{1}=\Delta_{m, n}^{\nu+1, \ldots, \nu+1, \nu, \ldots, \nu ; \mathbf{1}}$ of rook placements allowing at most $\nu+1$ rooks in the first $s$ rows, and at most $\nu$ rooks in the remaining $n-s$ rows. By Theorem 3.1 the complex $K_{1}$ is $\mu$-connected where $\mu=\min \{m-n-1, \nu n+s-2\}$. Consequently $K_{1}$ is $\mu$-connected where $\mu=\nu n+s-2$, provided the following equivalent of the inequality (8) is satisfied,

$$
m \geq n(\nu+1)+s-1
$$

Let us suppose that $n=p^{\alpha}$ is a prime power. The abelian group $G=(\mathbb{Z} / p \mathbb{Z})^{\alpha}$ acts without fixed points on the rows of the chessboard $[m] \times[n]$. By symmetrization of 
$K_{1}$ with respect to the group $G$ we obtain (Definition 2.1) the symmetrized multiple chessboard complex,

$$
\Sigma_{G}=\Sigma\left(\Delta_{m, n}^{\nu+1, \ldots, \nu+1, \nu, \ldots, \nu ; 1} ; G\right) .
$$

More explicitly $\Sigma_{G}=K_{1} \cup \cdots \cup K_{n}$ is the union of $n$ complexes where each $K_{i}$ is obtained from $K_{1}$ by the corresponding permutation $g \in G$, in particular the simplices of $\Sigma_{G}$ are rook placements on the chessboard $[m] \times[n]$ where up to $\nu+1$ rooks are allowed in some $s$ rows and at most $\nu$ rooks in the remaining $n-s$ rows. Which combinations are allowed is governed by the action of group $G$. Note that the group $G=(\mathbb{Z} / p \mathbb{Z})^{\alpha}$ acts without fixed points on the complex $\Sigma_{G}$.

We find it more convenient to symmetrize with respect to the full symmetry group so we focus our attention to the complex,

$$
\Sigma=\Sigma_{m, n}^{\nu+1, \ldots, \nu+1, \nu, \ldots, \nu ; \mathbf{1}}=\Sigma\left(\Delta_{m, n}^{\nu+1, \ldots, \nu+1, \nu, \ldots, \nu ; \mathbf{1}} ; S_{n}\right) .
$$

The action of the group $G=(\mathbb{Z} / p \mathbb{Z})^{\alpha}$ on the complex $\Sigma$ is fixed-point-free as before.

We are interested in estimating the connectivity of the complex $\Sigma=\Sigma_{m, n}^{\nu+1, \ldots, \nu+1, \nu, \ldots, \nu ; \mathbf{1}}$. Theorem 3.3 provides such a result and shows that $\Sigma$ has the same connectivity lower bound as the complex $K_{1}=\Delta_{m, n}^{\nu+1, \ldots, \nu+1, \nu, \ldots, \nu ; \mathbf{1}}$.

Theorem 3.3 Suppose that,

$$
\Sigma=\Sigma_{m, n}^{k_{1}, \ldots, k_{s}, k_{s+1}, \ldots, k_{n} ; \mathbf{1}}=\Sigma_{m, n}^{\nu+1, \ldots, \nu+1, \nu, \ldots, \nu ; \mathbf{1}}
$$

is the symmetric multiple chessboard complex obtained by the $S_{n}$-symmetrization of the multiple chessboard complex $K_{1}=\Delta_{m, n}^{\nu+1, \ldots, \nu+1, \nu, \ldots, \nu ; \mathbf{1}}$ where $k_{1}=\ldots=k_{s}=\nu+1$ and $k_{s+1}=\ldots=k_{n}=\nu$. Assume that the following inequality (inequality (91)) is satisfied,

$$
m \geq n(\nu+1)+s-1
$$

Then the complex $\Sigma$ is $\mu$-connected where,

$$
\mu=k_{1}+\cdots+k_{n}-2=\nu n+s-2
$$

Proof: By Theorem 4.2 the complex

$$
\Sigma=\Sigma_{m, n}^{k_{1}, \ldots, k_{s}, k_{s+1}, \ldots, k_{n} ; \mathbf{1}}=\Sigma_{m, n}^{\nu+1, \ldots, \nu+1, \nu, \ldots, \nu ; \mathbf{1}}
$$

is shellable. Since $\operatorname{dim}(\Sigma)=n \nu+s-1$ the complex $\Sigma$ is $(n \nu+s-2)$-connected and the result follows.

\section{Shellability of $\sum_{m, n}^{k_{1}, \ldots, k_{n} ; 1}$}

The following theorem was proved in JVZ-1. 
Theorem 4.1 For $m \geq k_{1}+k_{2}+\cdots+k_{n}+n-1$ the complex $\Delta_{m, n}^{k_{1}, \ldots, k_{n} ; 1}$ is shellable.

Here we show that the shelling order for the complex $\Delta_{m, n}^{k_{1}, \ldots, k_{n} ; 1}$, described in [JVZ-1, Section 4], can be in some cases extended to a shelling order on the symmetrization of $\Delta_{m, n}^{k_{1}, \ldots, k_{n} ; 1}$ with respect to the full symmetric group $\Sigma_{n}$.

Theorem 4.2 Suppose that,

$$
\Sigma=\Sigma_{m, n}^{k_{1}, \ldots, k_{s}, k_{s+1}, \ldots, k_{n} ; \mathbf{1}}=\Sigma_{m, n}^{\nu+1, \ldots, \nu+1, \nu, \ldots, \nu ; \mathbf{1}}
$$

is the symmetric multiple chessboard complex obtained by the $S_{n}$-symmetrization of the multiple chessboard complex $\Delta_{m, n}^{\nu+1, \ldots, \nu+1, \nu, \ldots, \nu ; 1}$ where $k_{1}=\ldots=k_{s}=\nu+1$ and $k_{s+1}=\ldots=k_{n}=\nu$. Assume the inequality $m \geq n(\nu+1)+s-1$ (the condition (9) in Section [3). Then the complex $\Sigma$ is shellable.

Proof: The dimension of the simplicial complex $\Sigma$ satisfies the inequality,

$$
\operatorname{dim}(\Sigma)+1=n \nu+s \leq m-n+1 .
$$

By construction the complex $\Sigma$ is the union,

$$
\Sigma=\bigcup \Delta_{m, n}^{a_{1}, \ldots, a_{n} ; \mathbf{1}},
$$

where $\left(a_{1}, \ldots, a_{n}\right)$ is an arbitrary permutation of $\left(k_{1}, \ldots, k_{n}\right)=(\nu+1, \ldots, \nu+1, \nu, \ldots, \nu)$. In other words $a_{1}+a_{2}+\cdots+a_{n}=n \nu+s \leq m-n+1, a_{i} \leq \nu+1$ for each $i$, and at most $s$ of the parameters $a_{i}$ can be equal $\nu+1$. Observe that as a consequence of Theorem 4.1 all of the constituent complexes $\Delta_{m, n}^{a_{1}, \cdots, a_{r} ; 1}$ of $\Sigma$ are shellable.

A facet of $\Sigma$ is naturally encoded as an $n$-tuple $\left(A_{1}, A_{2}, \ldots, A_{n}\right)$ of disjoint subsets of $[m]$, each of size $\leq \nu+1$, such that exactly $s$ of them have $\nu+1$ elements. We define the shelling order for the facets of $\Sigma$ by the following construction refining the construction from [JVZ-1].

$$
F=\left(A_{1}, A_{2}, \ldots, A_{n}\right) \text { is a predecessor of } F^{\prime}=\left(B_{1}, B_{2}, \ldots, B_{n}\right) \text { if either, }
$$

(a) $\left|A_{i}\right|=\left|B_{i}\right|$ for $i=1, \ldots, i_{0}-1$ and $\left|A_{i_{0}}\right|>\left|B_{i_{0}}\right|$, or

(b) $\left|A_{i}\right|=\left|B_{i}\right|=a_{i}$ for all $i=1,2, \ldots, n$ and in the shelling order of $\Delta_{m, n}^{a_{1}, \cdots, a_{n} ; 1}$ the simplex $F$ is a predecessor $F^{\prime}$.

Assume that $F=\left(A_{1}, A_{2}, \ldots, A_{n}\right)$ precedes $F^{\prime}=\left(B_{1}, B_{2}, \ldots, B_{n}\right)$ in the given order. We are looking for a facet $F^{\prime \prime}$ that is a predecessor of $F^{\prime}$ such that for a vertex $v$ of $F^{\prime}$,

$$
F \cap F^{\prime} \subseteq F^{\prime \prime} \cap F^{\prime}=F^{\prime} \backslash\{v\} .
$$

(a) If $\left|A_{i}\right|=\left|B_{i}\right|$ for $i=1, \ldots, i_{0}-1$ and $\left|A_{i_{0}}\right|>\left|B_{i_{0}}\right|$, then there exists $j>i_{0}$ such that $\left|B_{j}\right|>\left|A_{j}\right|$. Hence, there is a vertex $v$ in $B_{j}(v$ is a square $(x, j)$ in the $j$-th row) that does not belong to $A_{j}$. Let $B_{i_{0}}^{\prime}=B_{i_{0}} \cup\{x\}$ and $B_{j}^{\prime}=B_{j} \backslash\{x\}$. The facet $F^{\prime \prime}=\left(B_{1}, \ldots, B_{i_{0}}^{\prime}, \ldots, B_{j}^{\prime}, \ldots,\right)$ is clearly a predecessor of $F^{\prime}$. Moreover, the facets $F, F^{\prime}$ and $F^{\prime \prime}$ clearly satisfy the relation (13).

(b) If $\left|A_{i}\right|=\left|B_{i}\right|=a_{i}$ for all $i=1,2, \ldots, n$ then the existence of $F^{\prime \prime}$ and $v$ follows from the shelling of the complex $\Delta_{m, n}^{a_{1}, \ldots, a_{r} ; 1}$. 


\begin{tabular}{|c|c|c|c|c|c|c|c|}
\hline $\begin{array}{|ll|}\mathrm{F} & \\
& \mathrm{F}^{\prime}\end{array}$ & & & $\mathrm{F}$ & $\mathrm{F}^{\prime}$ & & $\begin{array}{|ll|}\mathrm{F} & \\
& \mathrm{F}^{\prime}\end{array}$ & $\mathrm{F}^{\prime}$ \\
\hline & $F^{\prime}$ & $\begin{array}{l}\mathrm{F} \\
\mathrm{F}^{\prime}\end{array}$ & & $\mathrm{F}$ & $\begin{array}{ll}\mathrm{F} & \\
& \mathrm{F}^{\prime}\end{array}$ & & $\mathrm{F}$ \\
\hline
\end{tabular}

Figure 2: The construction of $F^{\prime \prime}$ if $\left|A_{i}\right| \neq\left|B_{i}\right|$ for some $i$ (the case (a)).

\section{Proof of Theorem 1.2}

Theorem 5.1 (A. Volovikov [Vol-1]) Let $p$ be a prime number and $G=\left(\mathbb{Z}_{p}\right)^{\alpha}$ an elementary abelian p-group. Suppose that $X$ and $Y$ are fixed-point free $G$ spaces such that $\widetilde{H}^{i}\left(X, \mathbb{Z}_{p}\right) \cong 0$ for all $i \leq n$ and $Y$ is an $n$-dimensional cohomology sphere over $\mathbb{Z}_{p}$. Then there does not exist a G-equivariant map $f: X \rightarrow Y$.

Proof of Theorem 1.2; We begin with the observation that the general case of Theorem [1.2, corresponding to the pair of conditions,

$$
N \geq(r-1)(d+2) \quad r k+s \geq(r-1) d
$$

can be reduced to the case when the second inequality is actually an equality,

$$
N \geq(r-1)(d+2) \quad r k+s=(r-1) d
$$

Indeed, suppose that for given $d$ and $r$ one chooses a pair $\left(k^{\prime}, s^{\prime}\right)$ satisfying inequalities (14). Suppose that $(k, s)$ is the unique pair satisfying the equality $r k+s=(r-1) d$ and the condition $0 \leq s<r$ ( $s$ is the remainder of $(r-1) d$ on division by $r$ ). Then either $k<k^{\prime}$ or $k=k^{\prime}$ and $s \leq s^{\prime}$. In both cases the existence of a Tverberg $r$-tuple corresponding to the case (15) implies the existence a Tverberg $r$-tuple corresponding to the case (14) of the theorem.

Assuming (15) let us consider the simplicial complex,

$$
\Lambda=\Lambda(N, r ; k, s)=\bigcup \Delta_{N+1, r}^{k_{1}, \ldots, k_{r} ; \mathbf{1}}
$$

where exactly $s$ parameters $k_{i}$ are equal $k+2$ and all other parameters are $k+1$. This complex is evidently the configuration space of all possible choices for desired Tverberg partitions with the dimensions constrained as above. A moment's reflection reveals that $\Lambda$ is precisely the symmetric multiple chessboard complex $\Sigma_{m, n}^{\nu+1, \ldots, \nu+1, \nu, \ldots, \nu ; \mathbf{1}}$ from Theorem 4.2 for the following choice of parameters,

$$
m=N+1, \quad n=r, \quad \nu=k+1 .
$$

Following the usual reduction scheme [M03, Chapter] if a desired Tverberg $r$-tuple does not exist, there exists a $G$-equivariant map

$$
F: \Lambda \rightarrow\left(\mathbb{R}^{d}\right)^{* r}
$$


which misses the $d$-dimensional diagonal $\Delta \subset\left(\mathbb{R}^{d}\right)^{* r}$, where $G=\left(\mathbb{Z}_{p}\right)^{\alpha}$ acts without fixed points on both $\Lambda$ and $\left(\mathbb{R}^{d}\right)^{* r} \backslash \Delta$. Moreover, there is a $G$-equivariant homotopy equivalence, $\left(\mathbb{R}^{d}\right)^{* r} \backslash \Delta \simeq S^{D}$ where $D=(r-1)(d+1)-1$. By Theorem 5.1 (see also [M03, Section 6.2.6] for related results of other authors), this would lead to a contradiction if we are able to demonstrate that the complex $\Lambda$ is $D$-connected.

By Theorem 3.3 it is sufficient to guarantee the following pair of inequalities,

$$
m \geq n(\nu+1)+s-1 \quad \nu n+s-2 \geq(r-1)(d+1)-1
$$

for the choice of parameters given in (16). The second inequality in (17) is fulfilled in a tight way as the equality $k r+s=(r-1) d$. The first inequality in (17) is equivalent to the inequality,

$$
N+1 \geq r(k+2)+s-1=(r-1)(d+2)+1
$$

which is precisely the first inequality in (15). This observation completes the proof of the theorem.

\section{Constraint method and Sarkaria's inequality}

The symmetric, multiple chessboard complex played the key role in the proof of Theorem 1.2. The apparent incompatibility of the basic 'constraint method' (Section 6.1) with the symmetrized deleted join construction (Section 2.2), may be an initial explanation why Theorem 1.2 does not follow directly from either (TTT) or (OCTT) (see Section 1.2). In this section we review alternative 'indirect methods' and reduction procedures, which appear to be better adapted for the use of symmetrized deleted joins.

\subsection{Constraint method}

The proof of Theorem 7.2, based on Sarkaria's inequality, illustrates a method which may offer an interesting and useful alternative when the 'constraint method' [BFZ], (see also [Gr10, Section 2.9(c)] and [L02, Proposition 2.5]) is not directly applicable.

Recall that the 'constraint method' or the Gromov-Blagojević-Frick-Ziegler reduction, is an elegant and powerful method for proving results of Tverberg-van KampenFlores type. It can be summarized as follows.



Suppose that $K \subset L$ are two subcomplexes of the simplex $\Delta([n])=2^{[n]}$. Our objective is to prove that for each continuous function $f: K \rightarrow \mathbb{R}^{d}$ there exist disjoint faces $A_{1}, \ldots, A_{r}$ in $K$ such that $f\left(A_{1}\right) \cap \ldots \cap f\left(A_{r}\right) \neq \emptyset$. To this end we assume that $K$ is 
$(L, r)$-unavoidable in the sense that for each partition $A_{1} \uplus \ldots \uplus A_{r}=[n]$, where $A_{j} \in L$ for each $j$, at least one of the sets $A_{i}$ is in $K$.

Assume that such a statement is known to be true for each continuous map $F$ : $L \rightarrow \mathbb{R}^{d+1}$. An example is the simplex $L=\Delta^{N}$ (where $N=(r-1)(d+2)$ and $r=p^{k}$ is a prime power) by the 'topological Tverberg theorem' of Bárány, Shlosman, and Szücs [BSS], and Özaydin [Öz87] (see also [M03, Section 6.4]).

Extend the map $f: K \rightarrow \mathbb{R}^{d}$ to a map $\bar{f}(\bar{f} \circ e=f)$. Suppose that $\rho: L \rightarrow \mathbb{R}$ is the function $\rho(x):=\operatorname{dist}(x, K)$, measuring the distance of the point $x \in L$ from $K$. Define $F=(\bar{f}, \rho): L \rightarrow \mathbb{R}^{d+1}$ and assume that $\Delta_{1}, \ldots, \Delta_{r}$ is the associated family of vertex disjoint faces of $\Delta^{N}$, such that $F\left(\Delta_{1}\right) \cap \ldots \cap F\left(\Delta_{r}\right) \neq \emptyset$. More explicitly suppose that $x_{i} \in \Delta_{i}$ such that $F\left(x_{i}\right)=F\left(x_{j}\right)$ for each $i, j=1, \ldots, r$. Since $K$ is $(L, r)$-unavoidable, $\Delta_{i} \in K$ for some $i$. As a consequence $\rho\left(x_{i}\right)=0$, and in turn $\rho\left(x_{j}\right)=0$ for each $j=1, \ldots, r$. If $\Delta_{i}^{\prime}$ is the minimal face of $\Delta^{N}$ containing $x_{i}$ then $\Delta_{i}^{\prime} \in K$ for each $i=1, \ldots, r$ and $f\left(\Delta_{1}^{\prime}\right) \cap \ldots \cap f\left(\Delta_{r}^{\prime}\right) \neq \emptyset$.

For a more complete exposition and numerous examples of applications of the "constraint method' the reader is referred to [BFZ], see also [Gr10, Section 2.9(c)] and [L02, Proposition 2.5].

\subsection{The reduction based on Sarkaria's inequality}

In the 'direct approach' (Section [1.2) a map $f: K \rightarrow \mathbb{R}^{d}$ induces an $S_{r}$-equivariant map $\hat{f}: K_{\Delta}^{* r} \rightarrow\left(\mathbb{R}^{d}\right)^{* r} / \mathbb{R}^{d}$, where $\mathbb{R}^{d} \subset\left(\mathbb{R}^{d}\right)^{* r}$ is the diagonal subspace. The problem is to show that this map must have a zero, which is directly related to the problem of estimating the equivariant index $\left.\operatorname{Ind}_{G}\left(K_{\Delta}^{* r}\right) 1\right]$ This transition is functorial and in particular one obtains from (18) the following commutative diagram,

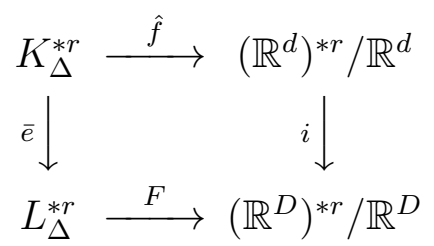

where $D=d+1$ (or more generally some other integer $D>d$ ). In this setting the constraint method, based on the diagram (18), can be replaced by the index calculation (Sarkaria's inequality) (based on the diagram (19)).

Recall that the Sarkaria's inequality [M03, Proposition 6.2.4] is the index inequality,

$$
\operatorname{Ind}_{G}(L) \geq \operatorname{Ind}_{G}\left(L_{0}\right)-\operatorname{Ind}_{G}\left(\Delta\left(L_{0} \backslash L\right)\right)-1
$$

where $L_{0}$ is a free $G$-complex, $L \subset L_{0}$ is $G$-invariant, and $\Delta\left(L_{0} \backslash L\right)$ is the order complex of the poset $\left(L_{0} \backslash L, \subseteq\right)$. By specializing $L_{0}=L_{\Delta}^{* r}$ and $L=K_{\Delta}^{* r}$ one obtains a tool for estimating the equivariant index of $K_{\Delta}^{* r}$.

\footnotetext{
${ }^{1}$ Here it is tacitly assumed, as in [M03, Section 6], that $G=\mathbb{Z}_{r}$, where $r$ is a prime number.
} 
The reduction based on Sarkaria's inequality is an interesting and useful alternative to the 'constraint method', which in some cases may facilitate calculation and lead to new observations. For illustration here is an extension of the "van Kampen-FloresGrünbaum-Schild non-embedding theorem', proved in [JVZ-2].

Theorem 6.1 Suppose that $r=p^{k}$ is a prime power. Let $K=K_{1} * \ldots * K_{s}$ where each $K_{i}$ is an $r$-unavoidable subcomplex ${ }^{2}$ of the simplex $\Delta^{m_{i}-1}=\Delta\left(\left[m_{i}\right]\right)$ spanned by $m_{i}$ vertices. Assume that the parameters $r, d, s$ and $\left\{m_{i}\right\}_{i=1}^{s}$ satisfy the inequality,

$$
(r-1)(d+s+1)+1 \leq m_{1}+\ldots+m_{s} .
$$

Then for each continuous map $f: K \rightarrow \mathbb{R}^{d}$ there exist $r$ disjoint faces $\Delta_{1}, \ldots, \Delta_{r}$ in $K$ such that $f\left(\Delta_{1}\right) \cap \ldots \cap f\left(\Delta_{s}\right) \neq \emptyset$.

\subsection{Extended Sarkaria's reduction}

The reduction based on Sarkaria's inequality is naturally extended to the case of symmetrized deleted joins (Section 2.2). This extension is especially interesting since it is not immediately clear how to establish a link with the 'constraint method'.

Suppose that $\mathcal{K}=\left\langle K_{1}, \ldots, K_{n}\right\rangle$ and $\mathcal{L}=\left\langle L_{1}, \ldots, L_{n}\right\rangle$ are two collections of simplicial complexes with vertices in $[m]$ such that $K_{i} \subseteq L_{i}$ for each $i$. In turn, there is an inclusion map $\mathcal{K}_{\Delta}^{* n}=K_{1} *_{\Delta} \ldots *_{\Delta} K_{n} \hookrightarrow \mathcal{L}_{\Delta}^{* n}=L_{1} *_{\Delta} \ldots *_{\Delta} L_{n}$ and the inclusion $e: \Sigma\left(\mathcal{K}_{\Delta}^{* n}\right) \hookrightarrow \Sigma\left(\mathcal{L}_{\Delta}^{* n}\right)$ of the associated symmetrized deleted joins (Section 2.2).

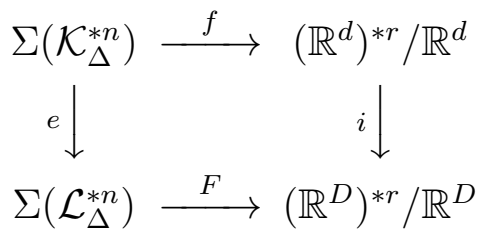

There is an associated commutative diagram (22) and the equivariant index of $\Sigma\left(\mathcal{K}_{\Delta}^{* n}\right)$ can be estimated from bellow by the inequality (20),

$$
\operatorname{Ind}_{G}\left(\Sigma\left(\mathcal{K}_{\Delta}^{* n}\right)\right) \geq \operatorname{Ind}_{G}\left(\Sigma\left(\mathcal{L}_{\Delta}^{* n}\right)\right)-\operatorname{Ind}_{G}(\Delta(Q))-1
$$

where $Q=\Sigma\left(\mathcal{L}_{\Delta}^{* n}\right) \backslash \Sigma\left(\mathcal{K}_{\Delta}^{* n}\right)$.

Propositions 6.2 and 6.3 are our first examples illustrating Sarkaria's reduction for symmetrized deleted joins. Suppose that $K \subset L \subset \Delta([m])=2^{[m]}$ are two simplicial complexes on the same set of vertices. For $1 \leq s \leq r$ let $\langle K, L\rangle_{s}^{r}=\langle K, \ldots, K, L, \ldots, L\rangle$ be the collection $\mathcal{K}=\left\langle K_{i}\right\rangle_{i=1}^{r}$ of $r$ complexes where $K_{i}=L$ for $i \leq s$ and $K_{j}=K$ for $j>s$. Such a collection is referred to as a $(K, L)$-collection of type $(r, s)$. If $\mathcal{K}=\left\langle K_{i}\right\rangle_{i=1}^{r}$ is a $(K, L)$-collection of type $(r, s)$ we denote the associated deleted join and symmetrized deleted join by,

$$
D J_{s}^{r}(K, L)=\mathcal{K}_{\Delta}^{* r} \quad S D J_{s}^{r}(K, L)=\Sigma\left(\mathcal{K}_{\Delta}^{* r}\right) .
$$

\footnotetext{
${ }^{2}$ Following [BFZ] and [JVZ-2] a complex $L \subset \Delta([k])$ is $r$-unavoidable if for each partition $A_{1} \uplus$ $\ldots \uplus A_{r}=[k]$ of the set of vertices there exists $i$ such that $A_{i} \in K$.
} 
Proposition 6.2 Assume that $1 \leq s<t<r$ where $r \geq 3$ is a prime number. Suppose that $\langle K, L\rangle_{s}^{r}$ and $\langle K, L\rangle_{t}^{r}$ are the associated $(K, L)$ collections of type $(r, s)$ and $(r, t)$. Then,

$$
\operatorname{Ind}_{G}\left(S D J_{s}^{r}(K, L)\right) \geq \operatorname{Ind}_{G}\left(S D J_{t}^{r}(K, L)\right)+s-t .
$$

Proof: In light of Sarkaria's inequality (23) it is sufficient to show that

$$
\operatorname{Ind}_{G}(\Delta(Q)) \leq t-s-1
$$

where $Q=S D J_{t}^{r} \backslash S D J_{s}^{r}$ and $S D J_{s}^{r}:=S D J_{s}^{r}(K, L), S D J_{t}^{r}:=S D J_{t}^{r}(K, L)$. For a simplex $a=\left(A_{1}, \ldots, A_{r}\right) \in S D J_{t}^{r}$ let $X_{a}:=\left\{i \in[r] \mid A_{i} \in L \backslash K\right\}$. Then $a \in Q=$ $S D J_{t}^{r} \backslash S D J_{s}^{r}$ if and only if

$$
s+1 \leq\left|X_{a}\right| \leq t
$$

Let $Q_{s}^{t}=\{Z \subset[r]|s+1 \leq| Z \mid \leq t\}$. Observe that $\operatorname{dim}\left(\Delta\left(Q_{s}^{t}\right)\right)=t-s-1$ and that the action of $G=\mathbb{Z}_{p}$ on $Q_{s}^{t}$ is free (here we use the fact that $t<r$ ).

The map $X: S D J_{t}^{r} \backslash S D J_{s}^{r} \rightarrow Q_{s}^{t}$ which sends $a=\left(A_{1}, \ldots, A_{r}\right)$ to $X_{a} \in Q_{s}^{t}$ is monotone and $G$-equivariant. By the monotonicity and the 'dimension axiom' of the index function (see properties (i) and (v) of [M03, Proposition 6.2.4]),

$$
\operatorname{Ind}_{G}(\Delta(Q)) \leq \operatorname{Ind}_{G}\left(\Delta\left(Q_{s}^{t}\right)\right) \leq t-s-1
$$

and the inequality (25) follows as an immediate consequence.

The condition $t<r$ limits the use of Proposition 6.2 in some situations. The following simple consequence of [M03, Proposition 6.2.4]) may be useful in such cases.

Proposition 6.3 Suppose that $\mathcal{K}=\left\langle K_{1}, \ldots, K_{n}\right\rangle$ and $\mathcal{L}=\left\langle L_{1}, \ldots, L_{n}\right\rangle$ are two collections of simplicial complexes with vertices in $[\mathrm{m}]$ such that $K_{i} \subseteq L_{i}$ for each $i$. If $Q=\Sigma\left(\mathcal{L}_{\Delta}^{* n}\right) \backslash \Sigma\left(\mathcal{K}_{\Delta}^{* n}\right)$ then

$$
\operatorname{Ind}_{G}\left(\Sigma\left(\mathcal{K}_{\Delta}^{* n}\right)\right) \geq \operatorname{Ind}_{G}\left(\Sigma\left(\mathcal{L}_{\Delta}^{* n}\right)\right)-\operatorname{dim}_{G}(\Delta(Q))-1
$$

\section{Tverberg A-P problem}

In this section we discuss the relation of Theorem 1.2 to the problem whether each admissible $r$-tuple is Tverberg prescribable. This problem, as formulated in [BFZ] and $[\mathrm{B}$, will be referred to as the Tverberg A-P problem or the Tverberg A-P conjecture.

Definition 7.1 ([BFZ, Definition 6.7]) For $d \geq 1$ and $r \geq 2$, an $r$-tuple $d=\left(d_{1}, \ldots, d_{r}\right)$ of integers is admissible if,

$$
\lfloor d / 2\rfloor \leq d_{i} \leq d \quad \text { and } \quad \sum_{i=1}^{r}\left(d-d_{i}\right) \leq d .
$$

An admissible $r$-tuple is Tverberg prescribable if there is an $N$ such that for every continuous map $f: \Delta_{N} \rightarrow \mathbb{R}^{d}$ there is a Tverberg partition $\left\{\sigma_{1}, \ldots, \sigma_{r}\right\}$ for $f$ with $\operatorname{dim}\left(\sigma_{i}\right)=d_{i}$. 
Question: (Tverberg A-P problem; [BFZ, Question 6.9.]) Is every admissible $r$-tuple Tverberg prescribable?

Note that in the Tverberg A-P problem the emphasis is on the existence (rather then the size) of $N$ which makes a given $r$-tuple admissible (Definition 7.1).

The balanced case of the Tverberg A-P conjecture is the case when the dimensions $d_{1}, \ldots, d_{r}$ satisfy the condition $\left|d_{i}-d_{j}\right| \leq 1$ for each $i$ and $j$. In other words there exist $0 \leq s<r$ and $k$ such that $d_{1}=\ldots=d_{s}=k+1$ and $d_{s+1}=\ldots=d_{r}=k$. In this case the second admissibility condition in (28) reduces to the inequality,

$$
r k+s \geq(r-1) d
$$

while the first condition is redundant. The case when all dimensions are equal $d_{1}=$ $\ldots=d_{r}$ is answered by the 'Generalized van Kampen-Flores theorem' [Sar, Vol-2, BFZ]. The following theorem extends this result to the case of all balanced $r$-tuples $d_{1}, \ldots, d_{r}$.

Theorem 7.2 Suppose that $r=p^{\kappa}$ is a prime power and let $\mathbf{d}=\left(d_{1}, \ldots, d_{r}\right)$ be a sequence of integers satisfying the condition $\left|d_{i}-d_{j}\right| \leq 1$ for each $i$ and $j$. Then if the sequence $\mathbf{d}$ is admissible (Definition 7.1) it is Tverberg prescribable.

Theorem 7.2 is an immediate consequence of Theorem 1.2. Here we offer another proof (in the case when $r$ is a prime) which illustrates the methods developed in Section 6.3, The second proof of the special case of Theorem 7.2 may be of some independent interest for at least two more reasons. It shows how one can avoid the use of Theorem 1.2 by reducing it to a the 'Generalized van Kampen-Flores theorem'. Moreover, it may open a possibility for testing some other instances of the general Tverberg A-P conjecture.

Proof of Theorem 3.1: ( $r$ is a prime number)

For $\nu=k+1$ and $1 \leq s \leq r$ let $\alpha_{s}$ be the $r$-sequence defined as follows,

$$
\alpha_{s}(i)= \begin{cases}\nu+1, & i=1, \ldots, s \\ \nu, & i=s+1, \ldots, r\end{cases}
$$

Let $\Sigma_{s}:=\Sigma_{m, r}^{\alpha_{s} ; \mathbf{1}}$ be the associated symmetric multiple chessboard complexes (Section 2.1). Observe that,

$$
\operatorname{dim}\left(\Sigma_{m, r}^{\alpha_{s} ; \mathbf{1}}\right)=r \nu+s-1=r(k+1)+s-1 \geq(r-1)(d+1)=\operatorname{dim}\left(\left(\mathbb{R}^{d}\right)^{* r} / \mathbb{R}^{d}\right)
$$

so in light of (22) and the condition (29), it is sufficient to establish the inequality,

$$
\operatorname{Ind}_{G}\left(\Sigma_{m, r}^{\alpha_{s} ; \mathbf{1}}\right) \geq r \nu+s-1
$$

This is certainly true if $s=r$. Indeed, this is the case when $\alpha_{1}=\ldots=\alpha_{r}=\nu+1$ and (32) is essentially the content of the 'Generalized van Kampen-Flores theorem'. Alternatively this inequality can be deduced as a direct consequence of Theorems 3.1 and 5.1 . 
For $s<r$ the inequality (32) is established by induction. Indeed, for $1<s \leq r$ the poset $Q_{s}:=\Sigma_{m, r}^{\alpha_{s} ; \mathbf{1}} \backslash \sum_{m, r}^{\alpha_{s-1} ; \mathbf{1}}$ is an antichain. It follows that $\Delta\left(Q_{s}\right)$ is 0-dimensional and from the Proposition 6.3 we deduce that,

$$
\operatorname{Ind}_{G}\left(\sum_{m, r}^{\alpha_{s-1} ; \mathbf{1}}\right) \geq \operatorname{Ind}_{G}\left(\sum_{m, r}^{\alpha_{s} ; \mathbf{1}}\right)-1
$$

so if the inequality (32) holds for a selected $s \leq r$ it is also true for $s-1$.

Remark 7.3 One can also use Proposition 6.2 to deduce the case $s=t<r-1$ of the inequality (32) directly from the case $s=r-1$. Indeed, this follows from the observation that $\Sigma_{m, r}^{\alpha_{s} ; \mathbf{1}}$ is a $(K, L)$-collection of the type $(r, s)$ where $K=\Delta([m])^{(k)}$ and $L=\Delta([m])^{(k+1)}$ are the respective skeletons of the simplex $\Delta([m])=2^{[m]}$.

\section{References}

[B] R. Bacher et al. 'An Erdös-Szekeres-type question', June 2011, http://mathoverflow.net/questions/67762/.

[BBZ] I. Bárány, P.V.M. Blagojević, G.M. Ziegler. Tverbergs theorem at 50: Extensions and counterexamples, Notices Amer. Math. Soc., August 2016, 732-739.

[BSS] I. Bárány, S.B. Shlosman, and A. Szűcs. On a topological generalization of a theorem of Tverberg. J. London Math. Soc., 23:158-164, 1981.

[BMZ] P.V.M. Blagojević, B. Matschke, G.M. Ziegler. Optimal bounds for the colored Tverberg problem. J. European Math. Soc., Vol. 17, Issue 4, 2015, pp. 739-754.

[BFZ] P.V.M. Blagojević, F. Frick, G.M. Ziegler. Tverberg plus constraints. B. London Math. Soc., 46:953-967, 2014.

[BZ] P.V.M. Blagojević, G.M. Ziegler. Beyond the Borsuk-Ulam theorem: The topological Tverberg story, arXiv:1605.07321 [math.CO].

[Gr10] M. Gromov. Singularities, expanders and topology of maps. Part 2: From combinatorics to topology via algebraic isoperimetry Geom. Funct. Anal. 20 (2010), 416-526.

[JVZ-1] D. Jojić, S.T. Vrećica, R.T. Živaljević. Multiple chessboard complexes and the colored Tverberg problem, arXiv:1412.0386 [math.CO]. (accepted for publication in J. Combin. Theory Ser. A.)

[JVZ-2] D. Jojić, S.T. Vrećica, R.T. Živaljević. Topology and combinatorics of 'unavoidable complexes', arXiv:1603.08472 [math.AT].

[JNPZ] D. Jojić, I. Nekrasov, G. Panina, R.T. Živaljević. Alexander r-tuples and Bier complexes, arXiv:1607.07157 [math.CO].

[J08] J. Jonsson. Simplicial Complexes of Graphs. Lecture Notes in Mathematics, Vol. 1928, Springer 2008. 
[KRW] D.B. Karaguezian, V. Reiner, M.L. Wachs. Matching Complexes, Bounded Degree Graph Complexes, and Weight Spaces of GL-Complexes. Journal of Algebra 239:77922001.

[L02] M. de Longueville. Notes on the topological Tverberg theorem, Discrete Math. 241 (2001) 207-233; and Discrete Math. 247 (2002) 271-297 (erratum).

[M03] J. Matoušek. Using the Borsuk-Ulam Theorem. Lectures on Topological Methods in Combinatorics and Geometry. Universitext, Springer-Verlag, Heidelberg, 2003. Corrected and updated second edition, Springer 2008.

[Öz87] M. Özaydin. Equivariant maps for the symmetric group. Unpublished manuscript, available online at http://minds.wisconsin.edu/handle/1793/63829, 1987.

[Sar] K.S. Sarkaria, A generalized van Kampen-Flores theorem, Proc. Amer. Math. Soc. 11 (1991), 559-565.

[Vol-1] A. Yu. Volovikov. On a topological generalization of the Tverberg theorem. Math. Notes, 59(3):324-326, 1996. Translation from Mat. Zametki 59, No.3, 454-456 (1996).

[Vol-2] A. Yu. Volovikov. On the van Kampen-Flores theorem, Math. Notes (5) 59 (1996), 477-481.

[VŽ94] S. Vrećica and R. Živaljević. New cases of the colored Tverberg theorem. In H. Barcelo and G. Kalai, editors, Jerusalem Combinatorics '93, Contemporary Mathematics Vol. 178, pp. 325-334, A.M.S. 1994.

[VŽ11] S. Vrećica, R. Živaljević. Chessboard complexes indomitable. J. Combin. Theory Ser. A, 118(7):2157-2166, 2011.

[Zi94] G.M. Ziegler. Shellability of chessboard complexes. Israel J. Math. 1994, Vol. 87, 97-110.

[Zi11] G.M. Ziegler. $3 N$ colored points in a plane. Notices of the A.M.S. Vol. 58 , Number 4, 550-557, 2011.

[ŽV92] R.T. Živaljević and S.T. Vrećica. The colored Tverberg's problem and complexes of injective functions. J. Combin. Theory Ser. A, 61(2):309-318, 1992.

[Živ98] R. Živaljević. User's guide to equivariant methods in combinatorics, I and II. Publ. Inst. Math. (Beograd) (N.S.), (I) 59(73):114-130, 1996 and (II) 64(78):107-132, 1998.

[Ž04] R.T. Živaljević. Topological methods. Chapter 14 in Handbook of Discrete and Computational Geometry, J.E. Goodman, J. O'Rourke, eds, Chapman \& Hall/CRC 2004, 305-330. 